\title{
Short Term Effects of Muscle Energy Technique vs. Active Release Technique in Improving Hamstring Flexibility and Pain in Patients with Acute Anterior Cruciate Ligament (ACL) Tear - A Randomized Control Trial
}

\author{
Vibhuti Vinodsingh Gaur ${ }^{1}$, Angela Arun Kapoor², Pratik Arun Phansopkar ${ }^{3}$ \\ 1,2, Department of Cardiorespiratory Physiotherapy, Ravi Nair Physiotherapy College, Datta Meghe Institute \\ of Medical Sciences, Wardha, Maharashtra, India. ${ }^{3}$ Department of Musculoskeletal Physiotherapy, Ravi Nair \\ Physiotherapy College, Datta Meghe Institute of Medical Sciences, Wardha, Maharashtra, India.
}

\section{ABSTRACT}

\section{BACKGROUND}

Hamstring tightness is a very commonly occurring condition. Poor hamstring flexibility is usually associated with injuries to the lower back and lower extremities. Active release technique and muscle energy technique both help in improving hamstring flexibility and pain in patients with acute anterior cruciate ligament (ACL) tear. While muscle energy technique (MET) is usually used by practitioners and manual therapists, there are only few studies supporting and accepting its use, as well as very few evidences to validate the theories used to elaborate the potency of muscle energy techniques. This has encouraged many researchers to find the benefits of other types of stretching on sport performance. The objective of this study is to compare the benefit of active release technique (ART) and MET in improving flexibility of hamstrings.

\section{METHODS}

60 subjects were divided into three groups. The muscle energy technique group included 5-minute warm-up followed by MET routine for 6 minutes and ART group included 5-minute warm up followed by an ART routine for 6 minutes. The subjects performed all the routines on three separate days.

\section{RESULTS}

Data analysis showed no significant difference ( $p<0.569$ ) in hamstring muscle flexibility following muscle energy techniques and active release technique types of stretching.

\section{CONCLUSIONS}

Both the techniques muscle energy technique and active release technique are equally effective.

\section{KEY WORDS}

Hamstring, Flexibility, Stretching, MET, ART, Muscle Force, Pain
Corresponding Author: Dr. Pratik Arun Phansopkar, Assistant Professor,

Department of Musculoskeletal Physiotherapy,

Ravi Nair Physiotherapy College, Datta Meghe Institute of Medical Sciences, Wardha, Maharashtra, India.

E-mail: drpratik77@gmail.com

DOI: $10.14260 /$ jemds/2021/29

How to Cite This Article:

Gaur VV, Kapoor AA, Phansopkar PA. Short term effects of muscle energy technique vs active release technique in improving hamstring flexibility and pain in patients with acute anterior cruciate ligament (acl) tear - a randomized control trial. J Evolution Med Dent Sci 2021;10(03):137142, DOI: $10.14260 / \mathrm{jemds} / 2021 / 29$

Submission 29-07-2020,

Peer Review 21-11-2020,

Acceptance 28-11-2020,

Published 18-01-2021.

Copyright (C) 2021 JEMDS. This is an open access article distributed under Creative Commons Attribution License [Attribution 4.0 International (CC BY 4.0)] 


\section{BACKGROUND}

Athletes perform warm-up exercises before any physical activities in order to enhance the performance and reduce the risk of any kind of muscular and bony injuries. ${ }^{1}$ There are lots of other advantages that can be achieved through warm-up like, increasing range of motion, delaying muscle fatigue, reducing and preventing delayed onset of muscle soreness, improving maximal muscle contraction, increasing the muscle and connective tissue temperature. ${ }^{2}$ Among all the different types of warm-ups, stretching was one of the most commonly followed warm-up routine ${ }^{3}$ However, there are several contrast opinions regarding the effectiveness of stretching. In contrast to the above ideas there has also been research that stated stretching increases range of motion ${ }^{2}$ and increase force and power production. ${ }^{4}$ In spite of these conflicting and contrast comments most athletes do include stretching in their warm-ups. ${ }^{3}$ The athletes are now aware of these issues and are practicing different types of stretching, thereby trying to minimize its harmful effect on sporting performance. This encouraged many researchers to find the benefits of different types of stretching on sport performance.

Most of the research on static stretching stated; it was detrimental to muscular performance ${ }^{5-9}$ but there are only few studies which investigated the effects of active release technique and dynamic stretching on athletic performance. Moreover, these researches showed conflicting findings. Young et al. (2001) found increased force production following active release technique stretching. ${ }^{1}$ In contrast, Church et al. (2001) found that active release technique stretching resulted in decreased of ACL injury. ${ }^{8}$ Similarly, Yuktasir et al. (2007) stated that active release technique stretching had no effect on force and power production. ${ }^{10}$

While MET is usually used by practitioners and manual therapists, there are only few studies supporting and accepting its use, as well as very few evidences to validate the theories used to elaborate the potency of muscle energy technique. Many researchers have investigated the benefit of contract-relax techniques (like MET) on hamstring flexibility and noted that contract-relax technique method have improved muscle flexibility 11,12 There by identified significant improvements seen in flexibility of hamstrings along with an increase in non-resistant force after a contract-relax stretching program. Some researchers claimed that contract-relax stretching were more beneficial than ballistic type of stretching for increasing muscle flexibility over a month period, though other researchers, however, have reported no significant differences between the two techniques, Madeleine \& Fryer (2008) found that both Chaitow and Greenman muscle energy technique improves flexibility immediately after the stretching, however no big variation between the two approaches. ${ }^{13,14}$ M. Waseem et al. (2009) stated that MET significantly increases the flexibility of muscle. ${ }^{15}$ As there is paucity in the literature, no such study is conducted on the comparison of both ART stretching and ME techniques on improving hamstring flexibility.

\section{METHODS}

A randomized control trial was conducted with sample size of 60 and the patients were randomly allocated into 20 participants in each group (Figure 1). Independent research assistants, blinded for patient characteristics, allocated patients to one of the intervention groups using a central computer-generated randomization scheme. Patients were recruited from OPD of musculoskeletal department, Ravi Nair Physiotherapy College, Sawangi (Meghe), Wardha.

The inclusion criteria were patient age between 20 to 40 years with acute ACL tear with or without reconstruction and subject willing to participate. The exclusion criteria was history of orthopedic problem, such as episodes of hamstring injury or fractures and surgery for other than ACL at knee joint in past 1 year, subjects with fixed flexion deformity.

The institutional ethical committee clearance was obtained from (IEC) Datta Meghe Institute of Medical Sciences (DMIMS). Permission from the principal of Ravi Nair Physiotherapy College was taken. Targeted population was selected from Musculoskeletal OPD, Ravi Nair Physiotherapy College (RNPC). The participants were selected according to criteria of inclusion and exclusion. Goniometer was used to measure the knee range of motion (ROM), measuring tape was used for measuring limb length and visual analog scale (VAS) scale was used for pain assessment. After getting informed consent, 60 patients were selected.

The active knee extension (AKE) test and sit \& reach test were performed before and after MET \& ART. The measurements of pre-test and posttest were noted accordingly. All the parameters were measured in degrees. Three consecutive readings were taken and the mean was recorded along with the age.

\section{Procedure}

Group A [Muscle Energy Technique (MET)]

MET was applied using post-isometric relaxation technique. The subject was in supine lying position and his hip was passively flexed and the leg extended until tightness was sensed by the examiner and till the subject reported a moderate stretching sensation. The participant provided a moderate (approximately 35 - $40 \%$ of maximal contraction) knee flexion isometric contraction, against the examiner's shoulder for 7 - 10 seconds. This was followed by $2-3$ seconds of relaxation and then the leg was passively stretched to the palpated barrier or tolerance to stretch and held for 30 seconds. The leg was then kept back on the plinth for a short resting period of about 8 - 10 seconds. For preventing the increase in blood pressure due to the maneuver ${ }^{14}$ and for reducing compensatory muscle recruitment during the isometric contraction, the subject was instructed to breathe normally and avoid hip elevation. This procedure was repeated two more times.

\section{Group B [Active Release Technique (ART)]}

With the knee in full extension, the partner gently pushed the subject's lower limb with the knee fully extended into hip flexion to the point of discomfort and foot in relaxed plantar flexion. The subject then was instructed to contract the antagonist (hamstrings) maximally into extension while being resisted by their partner for $5 \mathrm{~s}$. The subject then was instructed to maximally contract the agonist (quadriceps) into flexion for $5 \mathrm{~s}$ then relaxes; the partner then passively moved the leg further into flexion until discomfort and held for $20 \mathrm{sec}$. 
This procedure was repeated two more times with a 4 - 5 seconds rest period between each stretch. ${ }^{16}$

\section{Group C [Active Knee Extension Test (AKE)]}

The active knee extension test (AKE) was used to measure hamstring flexibility. The subject was instructed to lie in supine position and the non-affected limb and the pelvis was strapped to the couch for stabilization. The limb which was to be tested was positioned in 90 degrees of hip and knee flexion. Hip flexion was maintained through the use of a crossbar to maintain the proper position of hip and thigh ${ }^{17} \mathrm{~A}$ universal goniometer was used to measure the knee extension range of motion with appropriate placement. The subject was then asked to actively extend the knee, which is to be tested, as much as possible until a mild pain or stretch was felt. This procedure was done 3 times and the average was taken. The baseline, post and follow-up measurement data were collected from both groups.

\section{Sit and Reach Test}

The sit and reach test was performed using the procedures outlined in the American College of Sports Medicine (ACSM) manual 2. A standard sit and reach (SR) box was placed on the floor, by placing tape at a right angle to the $38 \mathrm{~cm}$ mark. The subject was told to sit on the floor with shoes on, and extend one leg up to full range of motion so that the sole of the foot was flat against the end of the box. The subject then extended the arms forward, placing one hand on top of the other. With palms facing down, the participant was told to reach forward sling hands along the measuring scale as much as possible without flexing the knee of the extended leg. Throughout the testing, it was checked to ensure that the heel remained at the $45 \mathrm{~cm}$ mark. Three repetitions were performed on one side and then the participant changed the position of the leg and repeated the same procedure with other side. The average of the three trials was taken for analysis.

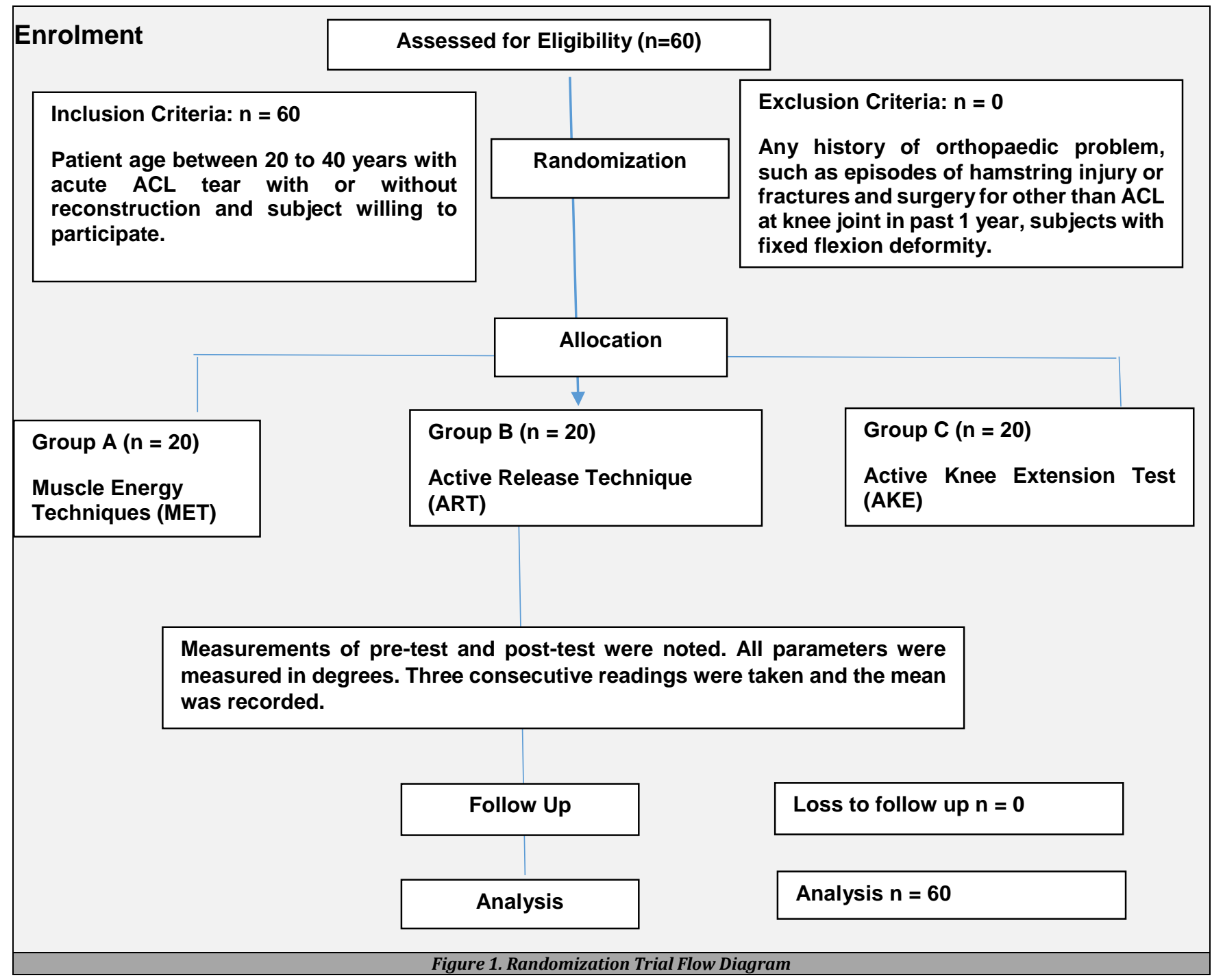

\section{Sample Size}

Sample size is determined using the following formula,

$N=2 X\left(\frac{z^{1-\frac{a}{2}}+Z_{1}-\beta}{\delta_{0}}\right)^{2} X S^{2}$

$N=\frac{2 x(1.96+6.45)^{2} x 6^{2}=16}{10}$
Where,

$\mathrm{N}=$ Size per group, $\mathrm{z}_{\mathrm{x}}=$ Standard normal deviate $=1.96$, 目 $=\mathrm{A}$ clinically acceptable margin, $\mathrm{S}^{2}=$ Polled standard deviation comparison groups.

Sample size per group estimated to 16 , including certain dropouts the sample size has been taken is 20 . The total minimum sample size with $95 \%$ of confidence interval is 60 
for 3 groups. The study is divided into three groups which will include 20 participants in each group.

\section{Statistical Analysis}

Analysis of the data was done by using descriptive and inferential statistics both. Descriptive statistics was used to describe the basic features of the data in a study and the inferential statistics was used to make inferences from our data to more general conditions. The statistical tests used for the analysis of the result were: one-way ANOVA and Post hoc test. Statistical analysis was performed using Statistical Package for the Social Sciences (SPSS) version 23.0. The statistical significant value for all analyses was set at $p \leq 0.05$.

\section{Data Analysis and Interpretation}

One-way ANOVA test verified the pretest values in the comparison between all the three groups on the basis of degrees of freedom.

\section{RESULTS}

\section{Active Knee Extension (AKE) Test}

There was a notable enhancement in post-stretch hamstring flexibility with muscle energy techniques of stretching (4.0\%) and there was a marked improvement in post-stretch with active release technique of stretching (3.3\%), while there were no significant changes in post-stretch with control group. It's also found that muscle energy technique is much effective than active release technique of stretching on muscle flexibility (Table 1). Following muscle energy technique of stretching, there was an increase in ROM $(2.7 \%, \mathrm{p}<0.05)$ immediately post stretching, when measured after 10 minutes the ROM further increased to $4.0 \%$, ( $<<0.05$ ). Following active release technique, there was an increase in ROM of knee $(2.0 \%, \mathrm{p}<0.05)$ immediately post stretching, when measured after 10 minutes the ROM further increased to $(3.3 \%$, p < $0.05)$. Statistics shows that there is noteworthy difference within the groups ( $p<0.066)$, but post hoc statistics for comparisons revealed that there was no marked difference in range of motion of knee between the two testing groups. Both testing groups showed notable advancements at post participation but the improvement in ME technique of stretching $(p<0.569)$ was more than that of AR technique of stretching ( $\mathrm{p}<0.961)$ (Graph 1, Table 3).

\begin{tabular}{|c|c|c|c|}
\hline Source of Variation & Sum of Squares & Degrees of Freedom & F Ratio \\
\hline Between Groups & 4.044 & 2 & \multirow{3}{*}{0.1465} \\
\hline Errors & 579.7 & 42 & \\
\hline Total & 583.8 & 44 & \\
\hline
\end{tabular}

\begin{tabular}{|cccc|}
\hline Source of Variation & Sum of Squares & Degrees of Freedom F Ratio \\
\hline Between Groups & 354.2 & 2 & 11.98 \\
Errors & 620.8 & 42 & \\
Total & 975.0 & 44 & \\
\hline Table 2. One Way ANOVA for Posttest Value of Hamstring Flexibility \\
between \& within All Groups
\end{tabular}

\begin{tabular}{|cccc|}
\hline Groups & $\begin{array}{c}\text { Age (in years) } \\
\text { Mean } \pm \text { SD }\end{array}$ & $\begin{array}{c}\text { Height (in cm) } \\
\text { Mean } \pm \text { SD }\end{array}$ & $\begin{array}{c}\text { Weight (in Kg) } \\
\text { Mean } \pm \text { SD }\end{array}$ \\
Group A (METs-PIR) & $17.6 \pm 1.1$ & $179.5 \pm 7.8$ & $71.6 \pm 9.3$ \\
Group B (ART) & $17.5 \pm 1.3$ & $176.5 \pm 6.5$ & $65.8 \pm 6.3$ \\
Control Group C & $19.7 \pm 1.1$ & $181.1 \pm 6.4$ & $80 \pm 8.7$ \\
\hline \multicolumn{4}{|c|}{ Table 3. Descriptive Data of Subjects in the Three Groups } \\
\hline
\end{tabular}

\begin{tabular}{|ccccc|}
\hline Duration & Group A & Group B & Group C & p Value \\
& Mean \pm SD & Mean \pm SD & Mean \pm SD & \\
$1^{\text {st }}$ day & $0.20 \pm 0.761$ & $0.07 \pm 0.254$ & $0.17 \pm 0.592$ & 3.221 \\
$2^{\text {nd }}$ day & $0.30 \pm 0.915$ & $0.17 \pm 0.407$ & $0.27 \pm 0.607$ & 5.274 \\
$3^{\text {rd }}$ day & $0.40 \pm 1.040$ & $0.27 \pm 0.450$ & $0.37 \pm 0.932$ & 0.389 \\
\hline $\mathrm{p}$ value & 0.023 & 0.037 & 0.028 & \\
\hline Table 4. Comparison of Vas Scores in Terms of Mean and Standard \\
Deviation of Different Stretching Techniques at Different Time \\
Intervals by One-Way ANOVA Test \\
\hline
\end{tabular}
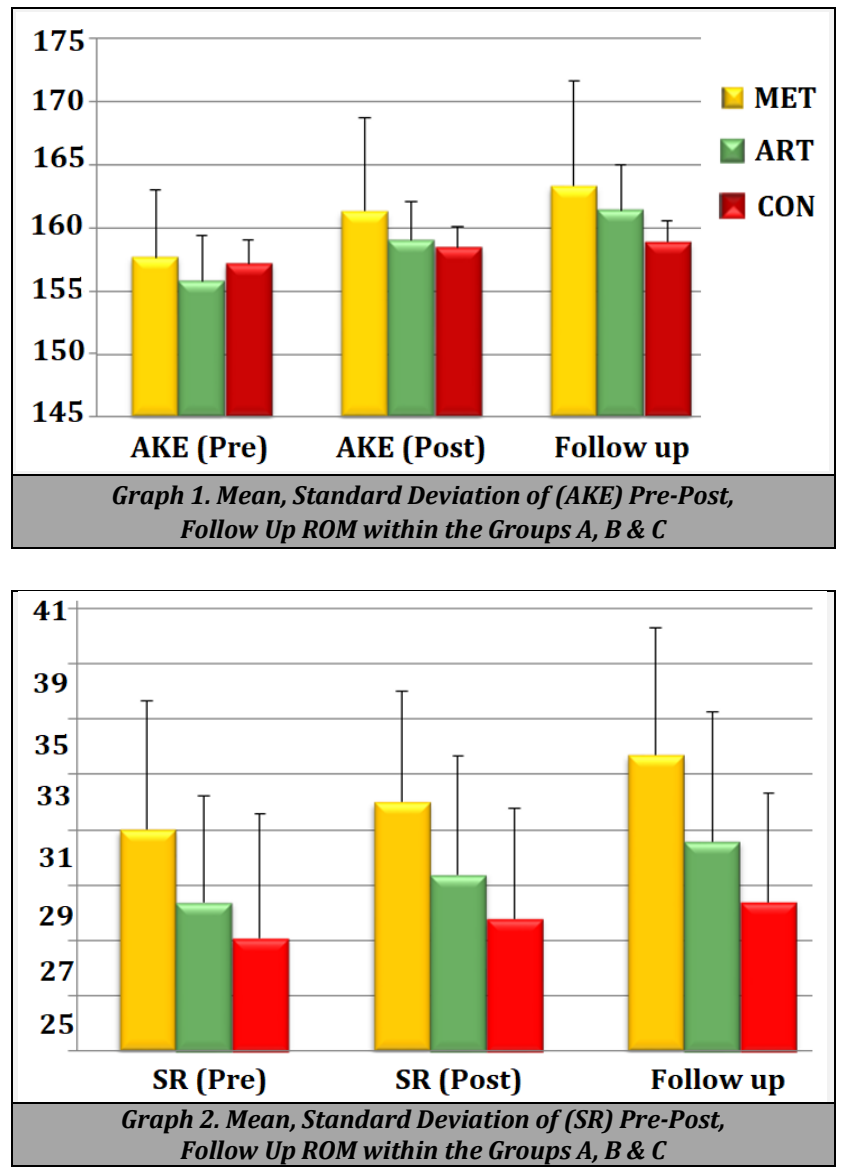

\section{Sit and Reach (SR) Test}

There was a marked improvement in post-stretch muscle flexibility with muscle energy techniques stretching (8.18\%, p $<0.05$ ). There was also some improvement in post-stretch of sit and reach measurements with ART stretching (5.26\%), while there was no such difference in post-stretch distance with control group. It's also found that muscle energy technique is much effective than the ART stretching on muscle flexibility. Following METs stretching, there was an increase in sit and reach measurement (3.03 \%, p < 0.05) post measurement, when measured after 10 minutes the ROM further increased to $(8.18 \%, \mathrm{p}<0.05)$ (Table 2). Following ART stretching, there was an increase in SR test $(3.02 \%, \mathrm{p}<$ 0.05 ) when measured after 10 minutes, the ROM further increased to $(5.26 \%)$. Statistics showed there is no marked difference within the groups also post hoc comparisons disclosed there were no major difference in ROM (knee) across 
the two testing groups (Graph 2). Both testing groups revealed significant advancement after involvement but the improvement in ME technique was better than that of ART stretching.

\section{DISCUSSION}

This study was a randomized controlled experimental design. Following the assessment of straight leg raising (SLR), participants were randomly divided into 3 equal groups, Group A (muscle energy technique (post isometric relaxation)), Group B (active release technique- contract relax agonist contract (CRAC)), Group C (control group). The treatment was given as one session in a day as all three groups' measurement took place in three different days. First day measurement i.e. the pretest was noted as baseline for all three groups; the testing group performed $3 \times 30$ second stretches with 10 - 15 seconds interval between stretches. Immediately just after the last stretch, participants were positioned for post-testing after 10 minutes. 10 minutes post-test measurements were taken according to previous study done by DePino et al. 18

The analysis of previous studies related to the role of various techniques in increasing muscle flexibility shows lots of information about the benefits. There were not many studies done before which compared the potency of two different stretching techniques i.e., ART and MET for improving hamstring flexibility and effects on muscle torque. This research was therefore conducted to compare the effectiveness of ME technique and ART stretching. For the aim of this comparison a pre-post test, follow up of both the experimental study group was carried out. Reason behind choosing the hamstring muscle is, hamstring muscle is the most frequent and most prone to injuries during the soccer games, and if the flexibility of hamstring muscle is adequate then possibilities of hamstring strain injuries can be reduced, and performance can be improved as well. Additionally, there are well recorded, reasonable and dependable techniques of testing hamstring flexibility, such as active knee extension test (AKE) and sit and reach (SR) test. A comparison of the pre and posttest and follow-up values of the ROM of the testing groups revealed that there was a notable advancement in experimental groups. Whereas no such improvement in controlled groups. Hence it can be said that the both the techniques are effective individually in increasing flexibility of hamstring muscles.

The current research used a 10-second cycle for the post isometric relaxation (PIR) technique and a 20 -second cycle for contract relax agonist contract (CRAC). The other shortest duration was a 30 seconds $\times 1 .^{19}$ followed by $4 \times 3,20$-second cycles; $^{20} 5 \times 3$, 20-second cycles; 21 and $5 \times 3$, 25-second cycles. $^{22}$ The aim for this research was to compare the effects of two different stretching techniques for increasing hamstring flexibility, to find the effects of muscle torque and reduce pain. The measurement tools used in this study to evaluate the hamstring muscle flexibility is active knee extension (AKE) and sit and reach (SR) test for the ROM and vertical Jump test for measuring the vertical jump performance of the muscle.
This research reveals that the hamstring flexibility remains notably improved after both stretching maneuver for 10 minutes. Previous study which was done using a static stretching protocol, the flexibility increased frequently but only stayed increased for 3 - 4 minutes after stretching. ${ }^{18}$ Spernoga et al. (2001) concluded his study, that "One-time, modified contract-relax stretching protocol was effective in improving hamstring flexibility as measured by active knee extension".22 However, the benefits of increased ROM lasted for only 6 minutes after the last stretch and thus this arrangement may not be any more beneficial than static stretching. In this study, there was a significant improving in both the experimental groups, marking that flexibility of the hamstrings can be increased effectively by both protocols. The research also reveals that post isometric relaxation (muscle energy technique) is more effective than contract relax agonist contract stretching for increasing hamstring muscle flexibility. The improvement in muscle energy techniques may be credited to the fact that pressure in the muscle creates autogenic inhibition over initiation of group Ib fibers, thus, causing muscle relaxation of the tight muscle. Additionally passive extension of the fascia and muscle as the movement of the joint occurs in the opposite direction after the muscle relaxes from max isometric contraction. The reason for the gain in flexibility can also be the change in stretch approach. Muscle energy techniques (MET) improve muscle length by a mixture of creep and plastic changes in connective tissue. It happens due to biomechanical change or neurological change or due to an increase in grit to stretching. Biomechanical and neuro-physiological mechanism might stimulate changes to range of motion and muscle tightness after the application of muscle energy techniques. ${ }^{23}$ The neurological factor is explained by inhibition of motor activity of muscle going to stretch, the purpose of the stretching is to reduce muscle activity to minimize resistance to stretching. Similarly, in this study both muscle energy technique and active release technique stretching showed marked difference in vertical jump height performance.

There was an overall (5.5\%) decrease in jump height performance immediately after METs and after 10 minutes and (4.5\%) reduction in VJP following ART stretching immediately and after 10 minutes. Magnusson et al. (2011) stated that "ART stretching induces stretch relaxation to the musculotendinous unit". 23 This results in improvement of muscle flexibility by decreasing the tautness in the tendon. This decrease in tautness could affect the force production in the muscular and tendinous unit because of increase in length, which does not have a positive benefit on the length-stretch relationship.1,4 This relationship depends upon the actin and myosin filaments. When a muscle fiber contracts from a point where there was only minimal overlap between actin and myosin filament, the force of muscle contraction is least. ${ }^{2}$ Similarly, in this study, both muscle energy technique and active release technique stretching could have increased the slack, hence increasing the length in the tendon and decrease the overlap between filaments, which could have resulted in decreased vertical jump height performance. Therefore, further studies are needed to know the effects of benefits of METs and ART stretching on vertical jump performance. 


\section{CONCLUSIONS}

The study reveals there is no notable difference in hamstring muscle flexibility following muscle energy techniques and active release technique of stretching. Both stretching protocols (MET and ART) are equally effective on increasing flexibility, muscle force and reduction in pain. The present research found no significant difference in muscle force after both stretching techniques between the test groups. Both MET and ART stretching are equally effective and beneficial in improving hamstring flexibility.

Data sharing statement provided by the authors is available with the full text of this article at jemds.com.

Financial or other competing interests: None.

Disclosure forms provided by the authors are available with the full text of this article at jemds.com.

\section{REFERENCES}

[1] Young W, Elliott S. Acute effects of static stretching, proprioceptive neuromuscular facilitation stretching, and maximum voluntary contractions on explosive force production and jumping performance. Res Q Exerc Sport 2001;72(3):273-9.

[2] Alter KJ. Book reviews. Comparative Political Studies 2004;37(1):121-4.

[3] Shrier I. Does stretching improve performance? A systematic and critical review of the literature. Clin J Sport Med 2004;14(5):267-73.

[4] Nelson NM, Wright A, Lowry RG, et al. Where is the theoretical basis for understanding and measuring the environment for physical activity? Environmental Health Insights 2008;2:111-6.

[5] McMillian DJ, Moore JH, Hatler BS, et al. Dynamic vs. staticstretching warm up: the effect on power and agility performance. J Strength Cond Res 2006;20(3):492-9.

[6] Power K, Behm D, Cahill F, et al. An acute bout of static stretching: effects on force and jumping performance. Med Sci Sports Exerc 2004;36(8):1389-96.

[7] Wallmann HW, Mercer JA, Mcwhorter JW. Surface electromyographic assessment of the effect of static stretching of the gastrocnemius on vertical jump performance. J Strength Cond Res 2005;19(3):684-8.

[8] Church JB, Wiggins MS, Moode FM, et al. Effect of warmup and flexibility treatments on vertical jump performance. J Strength Cond Res 2001;15(3):332-6.

[9] Bradley PS, Olsen PD, Portas MD. The effect of static, ballistic and proprioceptive neuromuscular facilitation stretching on vertical jump performance. J Strength Cond Res 2007;21(1):223-6.
[10] Yuktasir B, Kaya F. Investigation into the long-term effects of static and PNF stretching exercises on range of motion and jump performance. J Bodyw Mov Ther 2009;13(1):11-21.

[11] Gribble PA, Guskiewicz KM, Prentice WE, et al. Effects of static and hold-relax stretching on hamstring range of motion using the flexability LE1000. Journal of Sport Rehabilitation 1999;8(3):195-208.

[12] Handel M, Horstmann T, Dickhuth HH, et al. Effects of contract-relax stretching training on muscle performance in athletes. Eur J Appl Physiol Occup Physiol 1997;76(5):400-8.

[13] Smith M, Fryer G. A comparison of two muscle energy techniques for increasing flexibility of the hamstring muscle group. J Bodyw Mov Ther 2008;12(4):312-7.

[14] Chaitow L, DeLany J. Clinical application of neuromuscular techniques. Vol. 2. $2^{\text {nd }}$ edn. Churchill Livingstone 2011: p. 1-610.

[15] Waseem M, Nuhmani S, Ram CS, et al. A comparative study: static stretching versus eccentric training on popliteal angle in normal healthy Indian collegiate males. 2009;3(3):180-6.

[16] Brody LT, Hall CM. Therapeutic exercise: moving toward function. Wolters Kluwer/ Lippincott Williams \& Wilkins Health 2011: p. 779.

[17] Decoster LC, Cleland J, Altieri C, et al. The effects of hamstring stretching on range of motion: a systematic literature review. J Orthop Sports Phys Ther 2005;35(6):377-87.

[18] Depino GM, Webright WG, Arnold BL. Duration of maintained hamstring flexibility after cessation of an acute static stretching protocol. J Athl Train 2000;35(1):56-9.

[19] Sullivan MK, Dejulia JJ, Worrell TW. Effect of pelvic position and stretching method on hamstring muscle flexibility. Med Sci Sports Exerc 1992;24(12):1383-9.

[20] Worrell TW, Smith TL, Winegardner J. Effect of hamstring stretching on hamstring muscle performance. J Orthop Sports Phys Ther 1994;20(3):154-9.

[21] Osternig LR, Robertson RN, Troxel RK, et al. Differential responses to proprioceptive neuromuscular facilitation (PNF) stretch techniques. Med Sci Sports Exerc 1990;22(1):106-11.

[22] Spernoga SG, Uhl TL, Arnold BL, et al. Duration of maintained hamstring flexibility after a one-time, modified hold-relax stretching protocol. J Athl Train 2001;36(1):44-8.

[23] Waldén M, Hägglund M, Magnusson $H$, et al. Anterior cruciate ligament injury in elite football: a prospective three-cohort study. Knee Surg Sports Traumatol Arthrosc 2011;19(1):11-9. 